Journal of Computer Science 4 (12): 982-990, 2008

ISSN 1549-3636

(C) 2008 Science Publications

\title{
Discrete Simulation Framework for Wireless Cellular Networks
}

\author{
Maher Al-Sanabani, Shamala Subramaniam, Mohamed Othman and Zuriati Zukarnain \\ Department of Communication Technology and Networks, \\ Faculty of Computer Science and Information Technology, University Putra Malaysia, \\ 43400 Selangor, Malaysia
}

\begin{abstract}
The aim of the discrete simulation framework is to deal with issues of the next generation of wireless cellular networks (e.g., multimedia traffic, radio resource management and mobility... etc) and to provide a platform for current and future investigations involving wireless cellular networks. The simulator is built from the scratch to enable customization requirements of any research and to provide the freedom to understand, configure protocol modules, draw desired topologies and specifies the movement paths of the mobile users and to plot the necessary performance graphs. In order to evaluate and validate the performance of the designed simulator, a simulation model for the wireless cellular network and mobile environment deployed. The performance of the developed simulator compared with the performance characteristics of existing algorithms. Simulation results verify the developed simulator is able to recapture the results of both the numerical result and simulation results of the comparative system utilizing an idealistic platform. In addition the simulator incorporates detail algorithms for the correlated components between the entities of the cellular networks and the development of the discrete simulator.
\end{abstract}

Key words: Discrete simulation, wireless cellular network, performance modeling

\section{INTRODUCTION}

The growth in complexity of communication systems increases the time and effort required for analysis and design, the need to insert new technologies into commercial products quickly requires that the design be done in a timely, cost-effective and effortfree manner. These demands can be met only through the use of powerful computer-aided analysis and design tools. A large body of computer-aided techniques has been developed in recent years to assist in the process of modeling, analyzing and designing communication systems. The communication systems can be evaluated using formula-based calculations, waveform-level simulation or through hardware prototyping and measurements ${ }^{[1]}$.

With simulation-based approaches to performance evaluation, systems can be modeled with almost any level of detail desired and the design space can be explored more finely than is possible with formulabased approaches or measurements. With a simulationbased approach, one can combine mathematical and empirical models easily and incorporate measured characteristics of devices and actual signals into analysis and design. Simulated waveforms can also be used as test signals for verifying the functionality of hardware. Indeed, a simulation-based approach can be used to create a rapid prototyping environment for analysis and design of communication and signalprocessing systems, an environment in which software models can be combined with hardware data and real signals to produce designs that are timely, cost-effective and error-free. The primary disadvantage of the simulation approach is the computational burden, which can be reduced by a careful choice of modeling and simulation techniques.

Faced with this growing and complexity of the wireless communication, where the networks have grown too large to allow easy experimentation and too complicated to admit easy tractable mathematical analysis, network simulation has filled an increasingly important need, helping researchers and designers to understand the behavior and performance of complex networks and to understand the behavior exhibited by networking protocols not originally designed to operate in such environments ${ }^{[2,3]}$.

Simulation models can be further subdivided based on time characteristic ${ }^{[4]}$, as shown in Fig. 1. Static models represent a view where there is no concept of time. It is either immaterial or the model represents a

Corresponding Author: Maher Al-Sanabani, Department of Communication Technology and Networks, Faculty of Computer Science and Information Technology, University Putra Malaysia (UPM), 43400 Selngor, Malayisa 


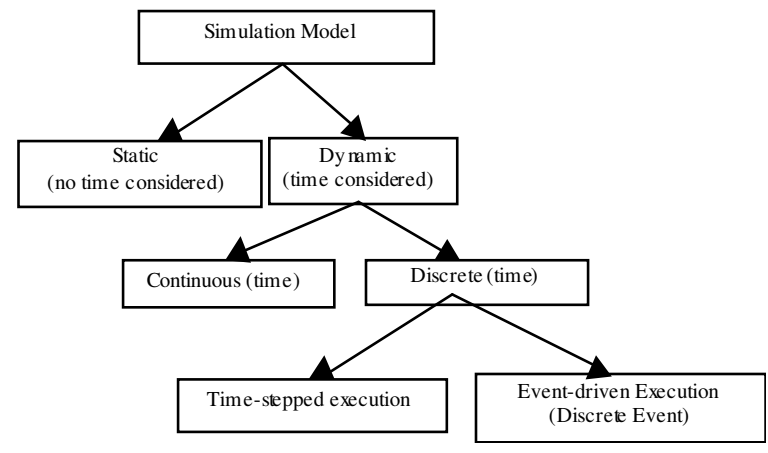

Fig. 1: Methods of Simulation Model

snapshot view of a time-changing system. For models that include time as a variable one usually differentiates between continuous time models and discrete time models, since these are treated differently. In a continuous time model, the system state is defined in all points of time, whereas it is only defined in certain discrete points in a discrete time model. Continuous time models are often described in terms of differential equations, where simulation consists of the numerical solution of these equations. Discrete time simulations are generally executed by having the computer perform discrete time changes to the system model state. If time is advanced in fixed steps of a given size, this is referred to as time stepped execution. The alternative is to specifically model the occurrence of significant events in the system and advance time with irregular steps between these events. This is referred to as eventdriven execution or discrete event simulation.

Although, there are many different simulators proposed in the literature that are currently being used by researchers to study the performance of mobile and fixed communication networks. Among the better known examples are: OPNET, OMnet++, NS-2, in particular. Although many of these efficient simulation tools exist, the motivation to build a simulator from scratch was to enable customization requirements of any research. Moreover, this model incorporates only the necessary assumptions for inclusion of the required parameters/functions for any investigation research. Some of the existing simulators do not provide a comprehensive operating specification, thus causing needs time to understand and have more complexity than necessary for the study. The advantage in developing a simulator is that it provides the freedom to understand, configure protocol modules, draw desired topologies, specifies the movement paths of the MTs and to plot the necessary performance graphs.

\section{DISCRETE SIMULATION FRAMEWORK}

From a functional point of view, Fig. 2 presents simulation framework architecture which has been proposed for wireless cellular network. The figure reflects the different procedures to be simulated, together with the interactions.

The inputs to the simulation framework are essentially the parameters that characterize the scenario to be evaluated. This includes the number and location of Base Station (BS) resulting from the radio network planning, the number of Mobile Terminal (MT) and services to be simulated as well as the service requirements and, finally, the specific parameters of the investigated algorithms under study. On the other hand, at the output, the simulation framework provides several performance indicators that allow the comparison among the different algorithms.

Figure 2 shows how the main core of the simulation framework contains the investigated algorithms to be evaluated. The operation of these algorithms is conditioned by the network model, the MT behavior in terms of mobility, traffic generation and the services requirements.

The network model allows specifying the network settings, the cell configuration and the layout to be studied according to the scenario defined in the planning. With respect to the mobility aspects, the different MTs are initially scattered in the scenario and follow trajectories that can be either deterministic or result from random mobility models. Depending on the trajectories and the network layout, the Received Signal Strength (RSS) is computed for every MT and BSs depend on the specific scenario. In the simulation framework, the RSS measurements are used by the handoff and cell selection algorithm to choose the serving cell for each MT.

With respect to traffic, note that the investigated algorithm are also conditioned by the traffic generation process. This involves deciding the instants when the MT start and finish the call are generated. Depending on the simulated services, different multimedia services with different requirement are used for this purpose.

The start of a new call will trigger an admission control algorithm to check if the MT can be accepted or rejected depending on the system status. If accepted call, admission control algorithm deals resource allocation algorithm. Similarly, the MTs in the neighbor cells that send a reservation request will trigger an admission control algorithm to check if the MT can be accepted or rejected depending on resource reservation algorithm and the system status. 


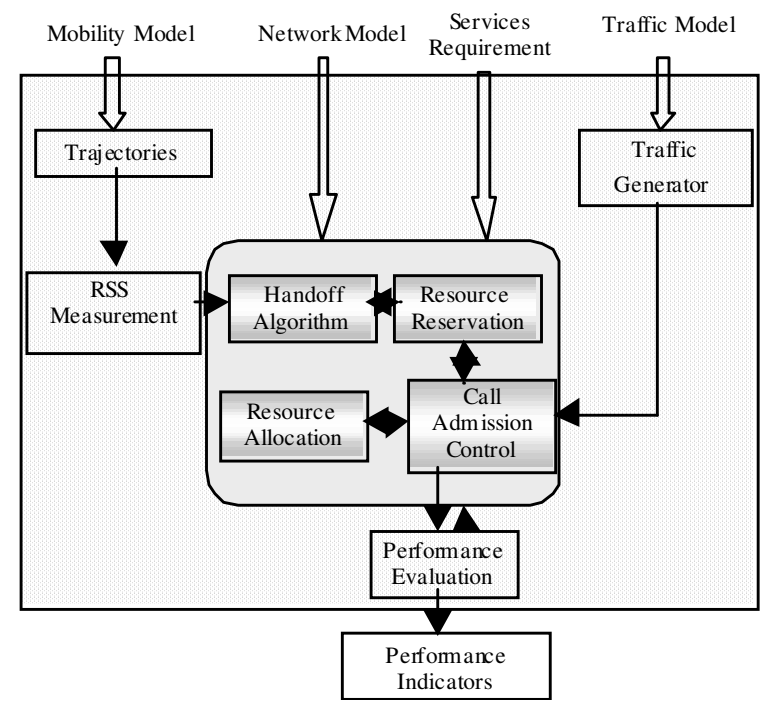

Fig. 2: Simulation framework

For a proper evaluation of the investigated algorithms, it is necessary to have simulation metrics that can provide distinct types of measurements and performance indicators and compile as much information as possible about the network behavior in order to find out what effects occur so that the influence of the investigated algorithm parameters can be better defined.

Network model: A simulation model for a wireless cellular network environment in two-dimensional (2-D) topology in which each cell has exactly six neighbouring cells is extensively developed based on ${ }^{[5]}$. The simulated area consists of Omni-cells and has a uniform geographic distribution. It is assumed that the BS is located in the center of a hexagonal. Each cell contains a BS, which is responsible for the connection setup and teardown of new connections and hand-off connections, as well as the reservation of bandwidth in neighboring cells. The cells are wrapped around so the topology of the simulated environment represents a sphere. This means that the handoff rates in all the cells are approximately similar. The graph in Fig. 3 shows a sample topology with wrapped around.

Mobility model: Mobility models are used to determine the trajectories that mobile terminals follow in the simulation framework. As in the case of propagation models, different mobility models are considered for different environments ${ }^{[6]}$.

A pseudo-random mobility model with semidirected trajectories ${ }^{[5,7,8]}$ is elaborated. In this model, the MTs are characterized by speed and direction and the

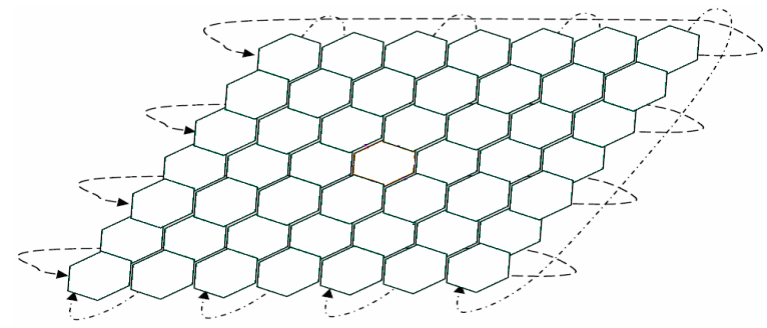

Fig. 3: Topology with wrapped around

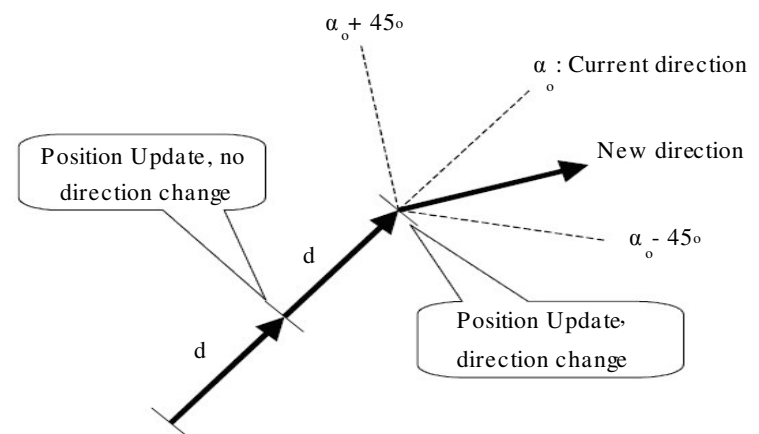

Fig. 4: Random Mobility Model with Semi-directed Trajectories

position is updated every $\mathrm{d}$ meters following the corresponding direction, where $\mathrm{d}$ is the length of distance which the MT must be move it before changing the direction, typically of $20 \mathrm{~m}$. At each position update, the direction is changed with probability 0.2 and in this case the angle for the direction change is selected randomly in the range $[+45,-45]$ degrees with uniform distribution. The process is illustrated in Fig. 4. At initialization, the MTs are scattered in the scenario and the MT direction is chosen randomly. The MT speed is kept constant, with typical values of 1 and $20 \mathrm{~m} \mathrm{sec}^{-1}$ for urban and suburban areas, respectively.

The Manhattan grid model whish is shown in Fig. 5. In this case, initially, the MTs are distributed randomly in the streets and move along them following straight trajectories. When a MT reaches a cross street, it turns with probability 0.5 and stays in the same street also with probability 0.5 . In the case of turning, the new direction is selected with equal probability (Fig. 5) ${ }^{[8,9]}$. The MT can only turn at the central point of the cross street. The MT position is updated every 5 meters and the speed can be changed at each position updated with probability 0.2 . The average speed is $1 \mathrm{~m} \mathrm{sec}^{-1}$ and a normal distribution with standard deviation $0.08 \mathrm{~m}$ $\mathrm{sec}^{-1}$ is assumed for speed changes with the constraint that the speed must be higher than $0 \mathrm{~m} \mathrm{sec}^{-1}$. 


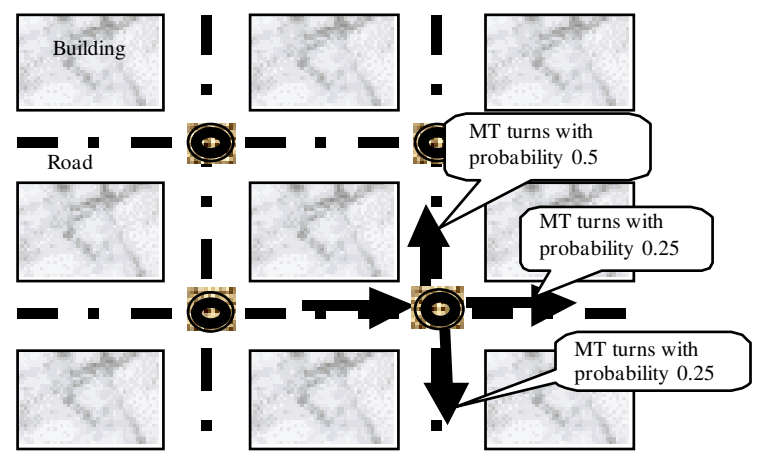

Fig. 5: Manhattan Grid Mobility Model

When a MT reaches the edge of the scenario, it is custom to apply the 'wrap-around' technique in which the MT enters through the opposite side of the edge that it is leaving, as if the scenario wrapped around on itself. By using the wrap-around technique, it is possible to collect statistics from all the cells in the scenario, reducing the simulation time needed to obtain statistically acceptable results. Instead, without wraparound, due to the border effect it is necessary to collect statistics only from central cells in the scenario, thus increasing the simulation time.

A different mobility models can be used based on the study.

Traffic model: The traffic model is the main feeder of the system. Call request of MTs are generated according to a Poisson process with average rate $\lambda$. All cells have the same arrival rate. Let $\mathrm{p}_{\mathrm{i}}$ be the probability of a call belonging to traffic class i $(i=1,2 \ldots \mathrm{k})$ where $\sum_{i=1}^{k} p_{i}=1$. Let $\lambda_{i}$ be the arrival rate of class $i$ at the cell. Then $\lambda \mathrm{i}=\lambda^{*}$ pi and total arrival rate is $\lambda=\sum_{\mathrm{i}=1}^{\mathrm{k}} \lambda_{\mathrm{i}}$.

New call from all the groups are generated with equal probability and appear anywhere in the cell with an equal probability. Two types of calls share the bandwidth of the cell: new calls and handoff calls.

The performance measures of the simulation results are plotted as a function of the call arrival rate. The call arrival rate is the arrival rate of new calls measured as the average number of new call requests per second per cell.

Multimedia services requirement: For serves multiple classes (multimedia traffic) of calls, the calls may differ in traffic characteristics (real time-Constant Bit Rate (rt-CBR), real time Variable Bit Rate (rt-VBR) and data traffic sources (non real time Unspecified Bit Rate-nrt-
UBR) and the desired Quality of Service (QoS) guarantees, i.e., delay bound, loss bound and throughput. In this research the promised traffic characteristics and the desired QoS guarantees can together be represented by a single number called effective bandwidth of the call. Note the concept of effective bandwidth in $^{[10]}$ is adopted from a wired network instead of a wireless network, where effective bandwidth means the minimum amount of bandwidth needed to provide a specific QoS given the traffic parameters of a call and the buffer size at the multiplexer. The fluid-flow analysis has been adopted $i^{[11]}$ for the mobile multimedia link layer to obtain an optimal bandwidth and code rate that can satisfy QoS parameters specified in terms of cell loss while maximizing the utilization of bandwidth . They named this bandwidth with code rate as wireless effective bandwidth. In the following discussions, when mentions a bandwidth requirements for each class of calls, it implies that the value of wireless effective bandwidth has been calculated using techniques have been discussed elsewhere $i^{[12]}$. We just make an assumption that the system will allocate a certain number of bandwidth units to different classes of calls.

A simulation deals with a broad range of multimedia services. The representations of various multimedia applications are based on the call duration, bandwidth requirement and class of service. The different application groups include rt-CBR, rt-VBR and nrt-UBR. These terms are borrowed from ATM networks literature. The source of data is reused from ${ }^{[13]}$ for a complete characteristic of the traffic. The six applications are carefully chosen for a simulation; which are typical traffic seen in wireless networks and their respective parameter values are chosen from Table 1 which is used recently in ${ }^{[14-16]}$.

The call duration is assumed to follow a geometric distribution between the minimum and maximum values and the bandwidth required for rt-CBR is fixed while rt-VBR and nrt-UBR is assumed to follow a geometric distribution between the minimum and maximum values.

New connections from all the application groups are generated with equal probability. The value closely represents realistic scenarios. The system has six priority levels and will assign one level for each type of traffic as shown in Table 1.

Resource allocation model: A Fixed Channel Allocation (FCA) scheme in each cell is assumed and the Complete Share (CS) strategy is adopted in this paper because this strategy gives better system utilization than the other strategies and less complex. 
Table1: Multimedia traffic characteristics

\begin{tabular}{|c|c|c|c|c|c|c|c|c|}
\hline \multirow{2}{*}{$\begin{array}{l}\text { Traffic } \\
\text { Type- No. }\end{array}$} & \multirow[b]{2}{*}{ Type of Media } & \multicolumn{3}{|c|}{ Bandwidth Requirements } & \multicolumn{3}{|c|}{ Duration of connection } & \multirow[b]{2}{*}{ Priority } \\
\hline & & Min & Avg. & $\operatorname{Max}$ & Min & Avg. & Max & \\
\hline rt-CBR-1 & Voice service and audio phone & $30 \mathrm{Kbps}$ & $30 \mathrm{Kbps}$ & $30 \mathrm{Kbps}$ & $1 \mathrm{~min}$ & $3 \mathrm{~min}$ & $10 \mathrm{~min}$ & 6 \\
\hline rt-CBR-2 & Video-phone and video-conference & $256 \mathrm{Kbps}$ & $256 \mathrm{Kbps}$ & $256 \mathrm{Kbps}$ & $1 \mathrm{~min}$ & $5 \mathrm{~min}$ & $30 \mathrm{~min}$ & 5 \\
\hline rt-VBR-3 & Interactive multimedia and video on demand & $1 \mathrm{Mbps}$ & $3 \mathrm{Mbps}$ & $6 \mathrm{Mbps}$ & $5 \mathrm{~min}$ & $10 \mathrm{~min}$ & $300 \mathrm{~min}$ & 4 \\
\hline nrt-UBR-4 & Email ,paging and fax & $5 \mathrm{Kbps}$ & $10 \mathrm{Kbps}$ & $20 \mathrm{Kbps}$ & $10 \mathrm{sec}$ & $30 \mathrm{sec}$ & $2 \min$ & 3 \\
\hline nrt-UBR-5 & Remote login and data on demand & $64 \mathrm{Kbps}$ & $256 \mathrm{Kbps}$ & $512 \mathrm{Kbps}$ & $30 \mathrm{sec}$ & $3 \mathrm{~min}$ & $10 \mathrm{~h}$ & 2 \\
\hline nrt-UBR-6 & File transfer and retrieval service & $1 \mathrm{Mbps}$ & $5 \mathrm{Mbps}$ & $10 \mathrm{Mbps}$ & $30 \mathrm{sec}$ & $2 \mathrm{~min}$ & $20 \mathrm{~min}$ & 1 \\
\hline
\end{tabular}

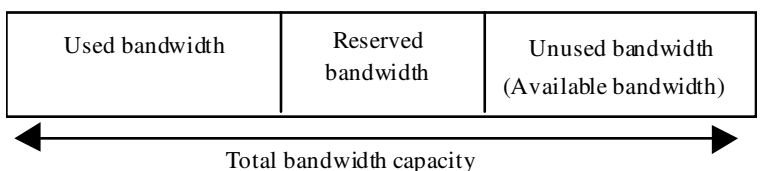

Fig. 6: Partitions of bandwidth in cell

The bandwidth allocation in the simulation for each cell is divided into three portions: the used, the reserved and the unused (available) which are illustrated in Fig. 6. The used bandwidth represents the portion of the cell bandwidth that is currently used by ongoing connections (it can be either new calls or handoff calls). The reserved bandwidth portion contains the bandwidth that is reserved by MTs for future handoff connections. The unused portion contains the bandwidth left over from the used and the reserved portion.

The simulation marking unused bandwidth from unused to use if the available bandwidth is sufficient to satisfy the new call while marking unused bandwidth from unused to reserve if the available bandwidth is sufficient to satisfy the handoff call for reservation. When the handoff is done, mark the reserved bandwidth from reserved to use. The sizes of used reserved and unused bands are varying size since at any time there is always used bandwidth released due to calls completion or handoff to another cell.

Three classes of traffic are assumed in this research: rt-CBR, rt-VBR and nrt-UBR. Real-time traffic includes voice service, video services, interactive multimedia and video-on-demand from users equipped with an adjustable rate $\operatorname{codec}^{[17-18]}$. In case of congestion, such users can gracefully adjust the coding rate such that the quality of video/audio received at destination(s) is still acceptable.

The bandwidth of a connection takes discrete values from the set $\left\{\mathrm{BW}_{\text {Min }}, \mathrm{BW}_{\mathrm{Avg}}, \mathrm{BW}_{\mathrm{Max}}\right\}$ have been assumed, Fig. 7 illustrates the case with one base value $\left(\mathrm{BW}_{\mathrm{Min}}\right)$ and two enhancement values $\left(\mathrm{BW}_{\mathrm{Avg}}, \mathrm{BW}_{\mathrm{Max}}\right)$. The $\mathrm{BW}_{\text {Min }}$ value that must be always be transmitted has bandwidth requirement while $\mathrm{BW}_{\mathrm{Avg}}$ and $\mathrm{BW}_{\mathrm{Ma}} \mathrm{X}$ values has more desirable bandwidth requirements.

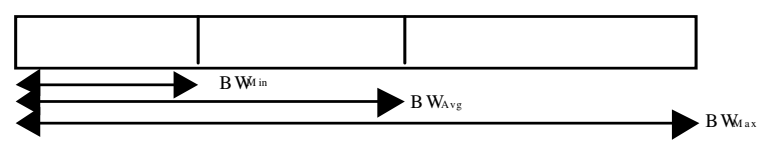

Fig. 7: Example of Levels of Bandwidth in Multimedia Stream

Table 2: General purpose languages vs. special purpose languages Advantages

- No additional effort required by modular to learn new programming language

- Less restriction on the formatting of output

- High flexibility in developing the model

Disadvantages

- Consume long programming time

- No automatic simulation debugging features

- No specific support for simulation modeling

- Higher programming error

Representation of models: The conceptual model must be translated into a representation suitable for a computer simulation. The representation of the system for the intended purpose, this raises the issue of selecting the appropriate simulation language. There are two main groups: general purpose languages and special purpose languages. Based on the advantages of general purpose over special purpose languages that are summarized in Table $2^{[19]}$, in this study, the simulation will be developed using a general purpose $\mathrm{C}++$ language.

The cellular model can built using a simulator developed specially for this purpose or using a commercial simulator. Purpose-built simulators are faster in terms of execution time, but the development time can be prohibitive. Commercial simulators are not as fast as Purpose-built simulators, because their code is much heavier than a single purpose simulator, but on the other hand, the existence of library models allows the faster construction of more detailed models.

Depending on the management of advancing time in the simulation, there are two types of modeling approaches: Time-Stepped simulation and EventDriven simulation as shown in Fig. 2. Based on the advantages of Time-Stepped over Event-Driven 
Table 3: Time-stepped vs. event-driven execution

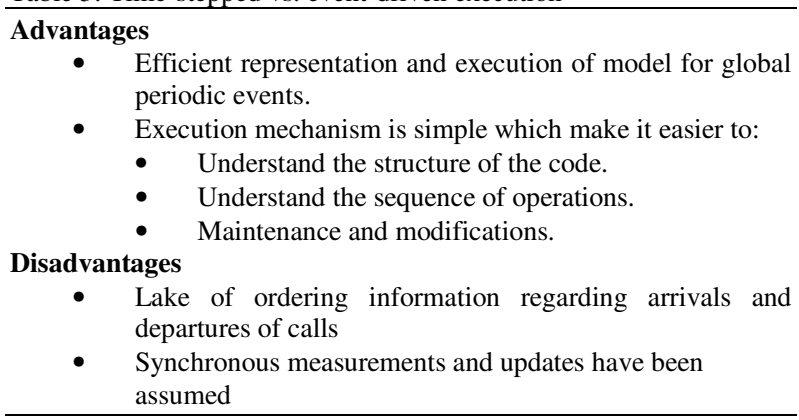

Execution that is summaries in Table 3. In this paper, the simulation will developed using a Time-Stepped modeling.

\section{DESIGN OF SIMULATOR MODEL}

The discrete simulator that is developed and used to perform all the performance evaluation experiments is referred to as the Wireless Cellular Network Simulator (WCNS). The simulator is written in $\mathrm{C}++$. The WNCS simulator evaluates the behavior of the investigated algorithms in multi-cell, multi-user and multi-service scenarios resulting from the previous network planning.

The WCNS include adequate modeling of the relevant aspects that have an impact on the performance of the strategies that are being evaluated. In the context of this research evaluation, these aspects include the traffic generation, the MT mobility and resource allocation taking into account that the research problem in wireless cellular network systems is of an inherent dynamic nature, i.e., the radio resource consumption by a given MT varies as the MT moves and depends on the MT behavior during a call.

The interaction between radio network planning and WCNS simulators is shown in Fig. 8. The WCNS is able to combine information about the network model with information about the position of the MTS and the traffic that they are likely to generate, in order to build a realistic picture of the network in terms of its coverage and the offered QoS. MTs are scattered around the network based on an expected traffic distribution. The WCNS is dynamic simulation because the MTs move around and generate traffic, so they behave as much as possible like real MTs. Consequently, the WCNS allows to capturing of the real situations and can provide more accurate results.

WCNS simulator structure: The core database of the simulator maintains two modeling the cells and MTs. Each of these models is connected to specific events

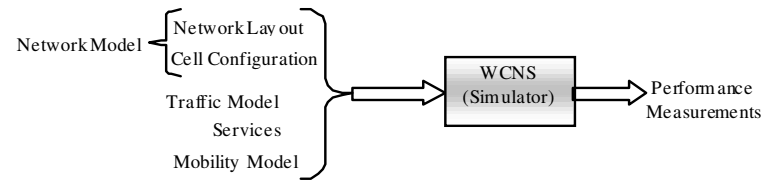

Fig. 8: Interaction between radio network planning and simulator

the cell model controls the new call arrival event whereas the mobile controls the handoff and termination event. Apart from the above main events, there exist bandwidth allocation events that are associated with each cell model. The allocation event calls procedures that determine how to allocate reserved or cellular resources to active calls inside a cell. Furthermore, each model also maintains specific performance related statistics. The more details of these models and events are described below.

Cell model: Each cell has an id that is an index to the global cell model database that is termed as CellMap. The CellMap keeps the knowledge about how the cells are connected (directional information) and captures hierarchical structure where cells can be a part of a region or cluster. Using the CellMap and cell id, one can find the adjacent cell ids in different direction. Therefore the CellMap is used to find the next cell to handoff a MT.

Mobile terminal model: Each MT has an id which is assigned when the MT is admitted and inserted in to the global MT database. Similarly, when a MT is terminated, its id is deleted from the MT database. As opposed to the cell model, the MT model is required to implement MT related properties and collect performance statistics that pertains to an individual MT's achieved performance. The MT model consists of the following classes of information.

Simulator events: The WCNS is encompasses a wide spectrum of QoS provisioning algorithms. As in discrete simulators, the integral or essence of the WCNS is the need to define events and their respective correlations. The main events and their interaction are as follows:

Arrival Event: Specific procedure related to generate a time to determine when the MT tries to make a call.

Call admission: User can implement specific call admission control procedure. This procedure will be executed by all cells when the call admission event occurs. All cell related information can be accessed by this procedure. 
Hand-in control: specific procedure for hand-in control determines what should be done when an existing call has hand-in to the current cell. Example of such procedure maybe modifying bandwidth allocation, updating statistics related to the handover calls.

Hand-out control: Specific procedure for hand-out control determines what needs to be done when a call has left the current cell. Example of such procedure may consider re-adjusting the bandwidth allocation to existing user, update available resources and update statistics related to handover call.

Call-termination control: Specific procedure related to call termination is called by a MT when a call termination event occurs. Example of such procedure may include updating different statistics related to the achieved QoS of the call.

Bandwidth allocation control: This procedure determines the bandwidth that should be allocated to different calls in each cell. This procedure is called when the allocation event occurs.

Bandwidth reservation: This procedure is related to one of the other events such as call admission or handoff event. This procedure allows for making bandwidth reservation.

Performance Metrics: In this simulation, three important performances metrics defined in cellular system traffic are as follows:

Handoff Connection Dropping Probability (HCDP): It is the ratio of the number that an ongoing call is dropped due to handoff failure because insufficient resources (all the resources in the target cell are busy), to the total number of handoff call roaming into that cell.

New Connection Blocking Probability (NCBP): It is the ratio of the number of blocked (non-accepted initially) new call requests due to the lack of available (insufficient) resource for this kind of requests, to the total of new call requests initiated inside the cell.

Bandwidth utilization: This metric represents the ratio of bandwidth used by serviced calls to the total capacity of the cellular system resources.

Case Study: Consider the uplink operation in a cellular network. A MT makes a call request to the system. Upon receiving the call request, the call admission control at BS makes a decision about whether its call

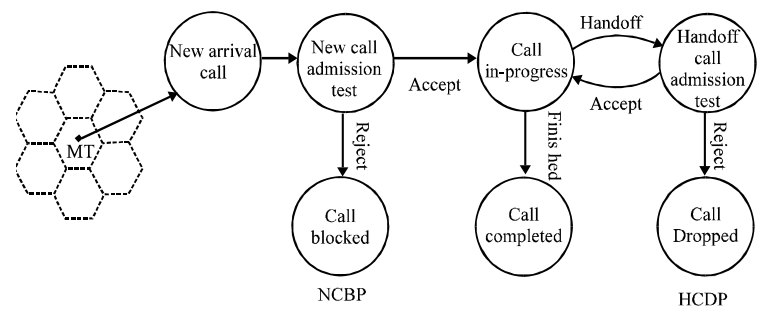

Fig. 9: State diagram of call

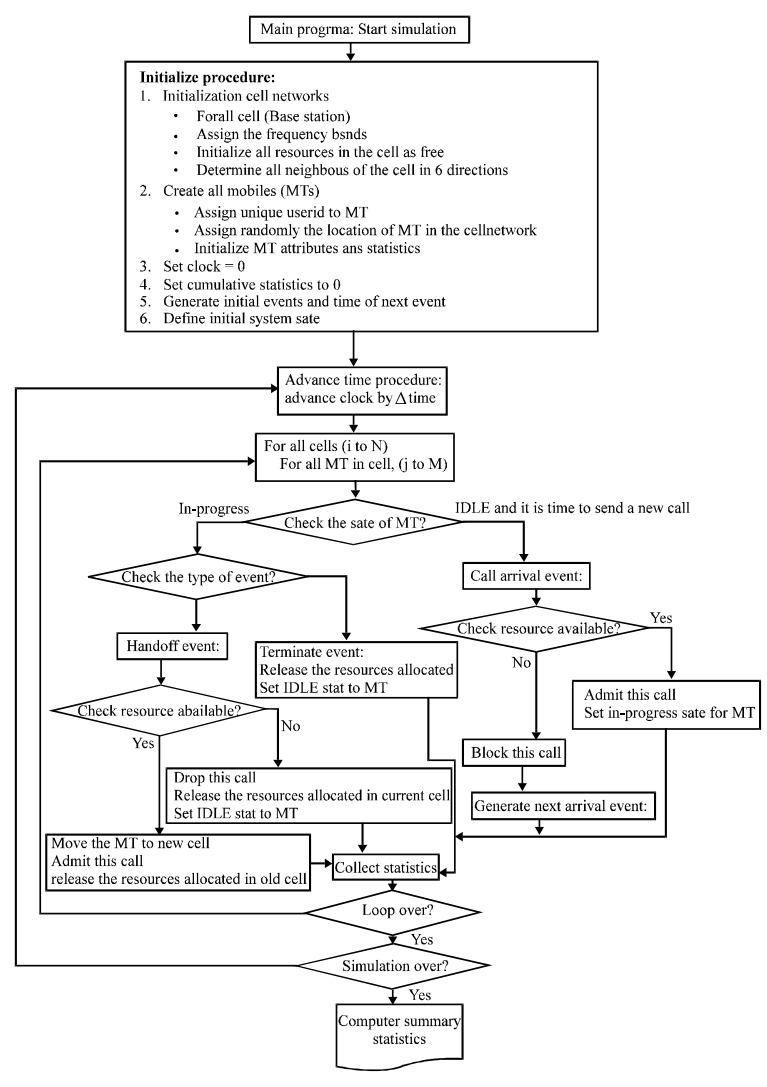

Fig. 10: The WCNS simulator flowchart

can be admitted or not. The admission decision is made on the declared traffic parameters and required QoS performance in the call request from the MT and the available resources in the system. The admission is then sent back from the BS to the MT which notified the MT about whether or not its call request can be accepted. If the admission decision is to accept, a call can be made between the MT and the BS before packet transmissions. Figure 9 shows the sate diagram of a call beginning from the arrived of a call.

Assume that a MT can be in any of the radio cells with equal probability when it originates a call. Each new call request is subject to a new call admission test 
at the BS. The failure of the test, results in call blocking and the probability of this is defined as NCBP. After successfully passing the admission test, a call is established between the MT and the BS for packet transmission. During the lifetime of its cell, the MT may move from place to place. When it migrates from the coverage area of one of the BS to that of another, handoff occurs. Whether or not the new BS can accept the call is subject to another admission test for the handoff call. Failure of this test, results in call dropping and the probability of this is defined as HCDP. A detail flowchart of the developed simulator is shown Fig. 10.

\section{RESULT AND DISCUSSION}

Simulator verification is an important part of any simulation study. In this work two schemes have been selected as validation of the simulator ${ }^{[13,20]}$. The simulation parameters are selected to achieve identical representation of these schemes. This is to check the correctness of the function of individual network components as well the co-ordination of different activities between them, fundamentally, to reproduce the same result. The details of these schemes and their respective results are as follows:

Model one description: The Adaptive Bandwidth Reservation (ABR) scheme ${ }^{[13]}$ provides call admission control and adaptive bandwidth reservation to guarantee QoS.

The results presented in Fig. 11 proofs that the WCNS simulator reproduce the results of the benchmark of ABR scheme from low to heavy traffic load distributions. Fig. 11 shows the NCBP and HCDP of real time data traffic services. The HCDP have similar trends, but due to the strict real time data call admission of the ABR scheme, NCBP for real time data increases rapidly in heavy traffic load situations.

From the simulation results obtained, it is proven that the WCNS simulator is able to recapture the results of simulation results of the base work. Figure 12 shows bandwidth utilization for the models. For low call arrival rates, the bandwidth utilization is virtually the same for the both. However, as the call arrival rate increases, the bandwidth utilization of WCNS simulator and ABR scheme has the same trends but with a variation of $11 \%$. The deviation is due to the random factor used in the simulation.

Model two descriptions: $\mathrm{In}^{[20]}$, a concept of Road Topology Based (RTB) mobility prediction technique is introduced to handle resource management issues caused by handoff. The RTB scheme in assist with

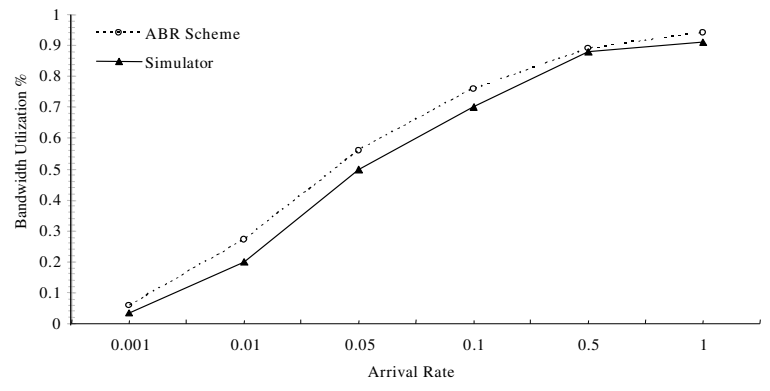

Fig. 12: Bandwidth Utilization vs. the Traffic Load

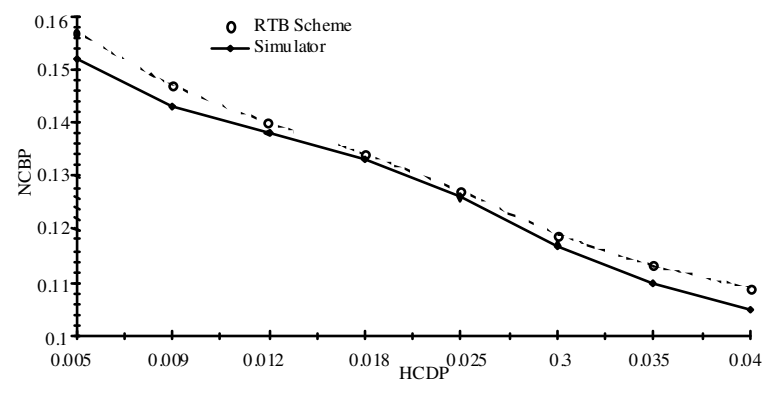

Fig. 12: Bandwidth Utilization vs. the Traffic Load

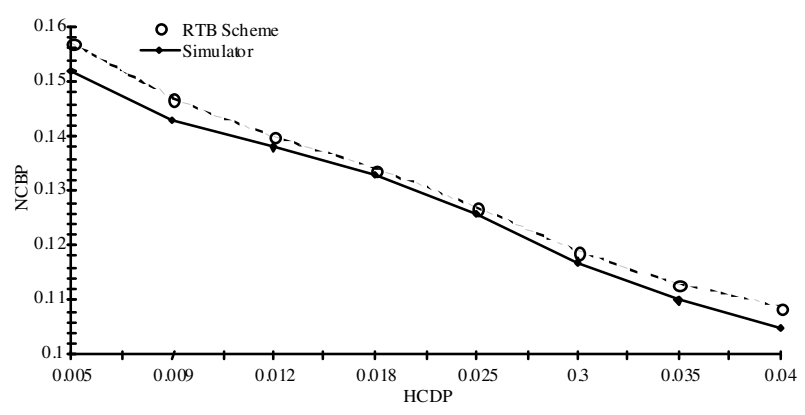

Fig. 13: NCBP vs. HCDP with Fixed Traffic Load $=1$

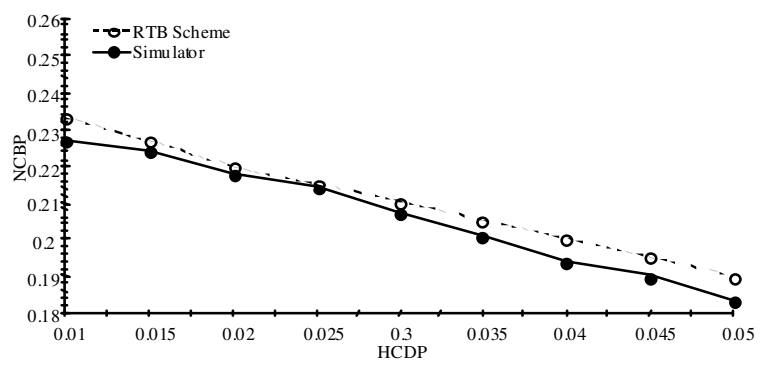

Fig. 14: NCBP vs. HCDP with Fixed Traffic Load $=1.2$

Global Position System (GPS) positioning capability provides accurate prediction on when and where the incoming and outgoing handoff events may occur. 
Thus, the system can reserve enough resource in advance.

The result of the comparative experiments is shows in Fig. 13-14. The results prove that the benchmark is achieved and the WCNS has encompassed all pertinent parameters.

\section{CONCLUSION}

A discrete simulation framework is developed for wireless cellular networks. The aim of the developed simulation framework was to provide a platform for future investigations involving wireless cellular networks. The WCNS has been designed to deal with important aspects for the planning and deployment of new services over existing network infrastructure and on the QoS level offered by cellular systems. The WCNS is able to recapture the results of both the numerical result and simulation results of previous work.

The simulation framework allows the modeling of different networks topologies and mobility. It can be easily extended to include new procedures that take into account new data and multimedia services as proposed by the advent of the next generation cellular networks. Also we can develop the simulator of cellular network by adding tool as a Graphical User Interface (GUI), which is used for parameters configuration and to construct a simulation of any topology, by just selecting, connecting and defining the properties of the network components.

\section{REFERENCES}

1. Jeruchim, M.C., P. Balaban and K.S. Shanmugan, 2002. Simulation of Communication Systems: Methodology, Modeling and Techniques. 2nd Edn., Kluwer Academic Publisher, USA.

2. Tranter, W.H., K.S. Shanmugan, T.S. Rappaport and K.L. Kosbar, 2003. Principles of Communication Systems Simulation with Wireless Applications. Prentice Hall Professional Technical Reference, USA.

3. Heidemann, J., Mills and S. Kumar, 2001. Expanding confidence in network simulations. IEEE Network, 15: 58-63.

4. Law, A.M., 2007. Simulation Modeling and Analysis. 4th Edn., McGraw-Hill, Boston.

5. Chiang, K.H. and N. Shenoy, 2004. A 2-D Random-Walk Mobility Model for LocationManagement Studies in Wireless Networks. IEEE T. Veh. Technol., 53: 413-424.

6. Tripathi, N.D., N.J.H. Reed and H.F. VanLandingham, 1998. Handoff in cellular systems. IEEE Wirel. Commun., 5: 26-37.

7. Lim, S., G. Cao and C. Das, 2004. A unified bandwidth reservation and admission control mechanism for QoS provisioning in cellular networks. Wirel. Commun. Mob. Com., 4: 3-18.
8. Markoulidakis, J.G., G.L. Lyberopoulos, D.F. Tsirkas and E.D. Sykas, 1997. Mobility modeling in third generation mobile telecommunications systems. IEEE Wirel. Commun., 4: 41-56.

9. Panzer, H. and R. Beck, 1990. Adaptive resource allocation in metropolitan area cellular mobile radio systems. Proceedings of $40^{\text {th }}$ IEEE VETC, pp: 638-645.

10. Kim, J.G. and M. Krunz, 1998. Effective bandwidth in wireless ATM networks. Proceedings of the 4th Annual ACM/IEEE International Conference on Mobile Computing and Networking, pp: 233-241.

11. Ramanathan, P., K.M. Sivalingam, P. Agrawal and S. Kishore, 1999. Dynamic resource allocation schemes during handoff for mobile multimedia wireless networks. IEEE J. Sel. Area Commun., 17: 1270-1283.

12. Cheng, Y. and W. Zhuang, 2003. Effective bandwidth of multiclass markovian traffic sources and admission control with dynamic buffer partitioning. IEEE T. Commun., 51: 1524-1535.

13. Oliveira, C., J.B. Kim and T. Suda, 1998. An adaptive bandwidth reservation scheme for highspeed multimedia wireless networks. IEEE J. Sel. Area Commun., 16: 858-874.

14. Sanabani, M., S. Shamala, M. Othman and Z. Zukarnain, 2007. Resource management at connection level for multimedia in wireless/mobile cellular networks. JMM, 3: 168-178.

15. Sanabani, M., S. Shamala, M. Othman and Z. Zukarnain, 2007. An enhanced bandwidth reservation scheme based on road topology information for qos sensitive multimedia wireless cellular networks. LNCS, Springer-Verlag, Berlin Heidelberg (ICCSA2007-MOBILECOMM.), 4706: 261-274.

16. Sanabani, M., S. Shamala, M. Othman and Z. Zukarnain, 2007. Multi-class bandwidth reservation scheme based on mobility prediction for handoff in multimedia wireless/mobile cellular networks. Wireless Pers. Commun, 46: 143-163.

17. Vickers, B.J., M. Lee and T. Suda, 1997. Feedback control mechanism for real-time multipoint video services. IEEE J. Sel. Area Commun., 15: 512-530.

18. Kanakis, H., P. Mishra and A.R. Reibman, 1995. An adaptive congestion control scheme for realtime video packet transport. IEEE/ACM $\mathrm{T}$. Network, 3: 671-682.

19. Robinson, S., 2004. Simulation: The Practice of Model Development and Use. John Wiley and Sons Ltd, England.

20. Soh, W.S. and H.S. Kim, 2006. A predictive bandwidth reservation scheme using mobile positioning and road topology information. IEEE/ACM T. Network, 14: 1078-1091. 